\title{
IMAGE-BASED IDENTIFICATION AND GIS-INTEGRATION OF VEHICLE RESTRAINT SYSTEMS AND EVALUATION OF SAFETY EFFECTS
}

\author{
CHRISTIAN STEFAN, CSABA BELEZNAI, ISABELA ERDELEAN \& MATTHIAS HAHN \\ AIT Austrian Institute of Technology, Austria.
}

\begin{abstract}
Collisions between vehicles leaving the road and unforgiving roadside objects such as trees, poles, road signs, etc. constitute a major road safety issue. On the Austrian road network, approximately 7.500 injury crashes occur every year due to run-off-road (ROR) manoeuvres (i.e. $20 \%$ of all injury crashes on public roads), contributing $35 \%$ to fatalities and $25 \%$ to serious injuries. Vehicle restraint systems (VRS) such as guardrails, concrete barriers, terminals or crash attenuators play a decisive role in mitigating the consequences of ROR crashes. Unfortunately, most national road administrations (NRA) do not have a centralized data management, while geo-referenced information on VRS and their safety-related attributes are also not available as digitized data. Researchers from the AIT have developed a novel approach to investigate, classify and evaluate VRS by means of image data processing, towards providing a comprehensive VRS inventory. The information obtained can be used for benefit-cost-analyses, road safety inspections and the evaluation of the effectiveness of different vehicle restraint systems.

Keywords: Asset management, database, decision tree, geographical information system, inventory control, run-off-road crashes, traffic safety, vehicle restraint system.
\end{abstract}

\section{INTRODUCTION}

\subsection{Analysis of road crashes}

Run-off-road crashes are extremely severe road accidents that often result in serious injuries or fatalities. Their relevance as a traffic safety issue has already been demonstrated in numerous European research projects (e.g. IRDES, RISER, SaVERS, SICHERE LEITUNG). The accident analysis conducted within the RISER project [1] highlights for example that even though only $10 \%$ of the total number of accidents are single vehicle accidents (typically associated to ROR crashes), the proportion of single vehicle events increase up to $45 \%$ when only fatal accidents are considered. The segment of fatalities resulting from this accident type varies from within European countries, but single vehicle accident fatalities constitute approximately one third of annual road fatalities [2].

According to data from 2012-2017, 7.537 ROR crashes occur every year on average on Austrian roads, representing $19.6 \%$ of all injury crashes on the public road network and contributing to $35.1 \%$ of all fatalities and $25.2 \%$ of all serious injuries on the road. Among the different types of ROR crashes, collisions with hazardous road objects (i.e. bridge pillars, lamp posts, trees) on the kerb side pose a major threat to vehicle passengers. According to Austrian accident statistics [3], the risk of death increases more than six-fold, if a vehicle running off the road hits a rigid object on the edge of the carriageway.

This is one of the arguments why the Austrian motorway operator ASFINAG states in its latest Road Safety Programme 2020 [4] that new safety strategies such as forgiving roadsides and self-explaining roads must be promoted, in order to prevent vehicles from running off the road and, in a worst-case scenario, collide with stationary obstacles on the roadside. 
The forgiving roadside concept is based on the principles of 'Vision Zero', advocating that driving errors should not automatically result in serious or fatal injuries [5], [10]. Its main focus is on measures that bring errant vehicles safely back into the lane to prevent ROR crashes in the first place. If a vehicle still hits a road element, the second priority of a forgiving roadside is to reduce the severity of the crash. Hence, vehicle restraint systems such as steel/concrete barriers or crash attenuators are key road infrastructure elements contributing to roadside safety. VRS are designed and constructed in such a way that vehicles running off the road will be contained and redirected to the carriageway with minimal damage to the passengers.

Whilst there are several international standards covering testing, evaluation and classification of vehicle restraint systems [6], [7], [8], their selection, location and installation requirements are typically based upon national guidelines and standards, often produced by National Road Authorities and/or overseeing organisations. Due to local conditions, these national guidelines vary considerably across the world (Fig. 1).

In order to identify safety critical road segments, it is necessary to know both the exact location and technical attributes of existing VRS on the road network. Yet, in most European countries VRS installations do not exist as digitally mapped assets, due to their age and the previous lack of technologies capable to perform large-scale digitization, mapping and analysis. From November 2018 till March 2019, the AIT conducted an online survey among European NRAs inquiring if detailed information concerning existing vehicle restraint systems on their respective road networks are readily available. This information should contain knowledge on safety-related performance indicators such as containment level, impact severity and working width, which define the functionality of a VRS according to EN 1317-2 [6].

Overall, 26 questionnaires were returned, 18 of which were fully completed and taken as a basis for analysis of the current status. $61 \%$ of the NRAs (11 votes) conceded to a total or partial absence of data regarding technical details of the installed VRS on their network. Yet, modern asset management as well as traffic safety evaluations rely on in-depth and highprecision data to guarantee an objective and comprehensive decision-making process.

Against this background, the study pursues two main objectives. First, a methodology for classifying vehicle restraint systems by means of image data processing is described (Chapter 2 .

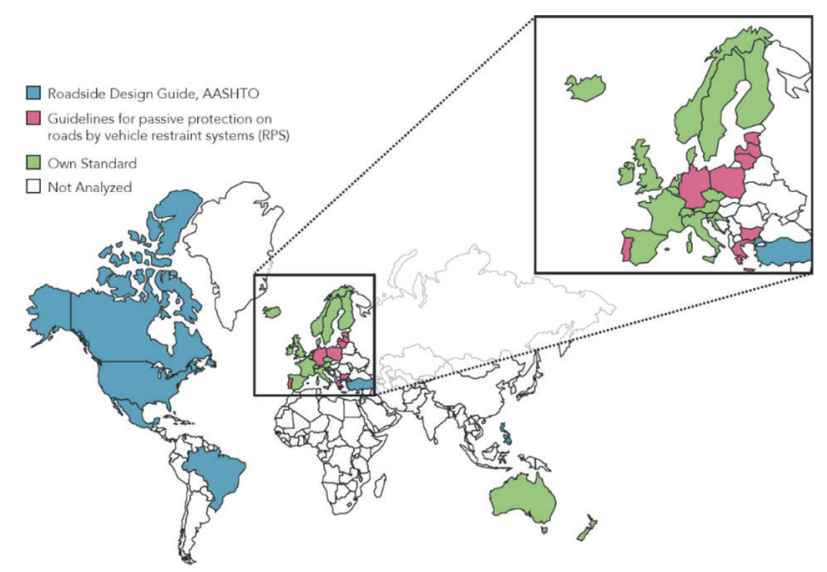

Figure 1: Country-specific VRS requirements and installation guidelines [9]. 
The information obtained was used to evaluate (i) what kind of VRS was installed in front of hazardous road objects and (ii) if the total length (so called Length of Need) of a barrier in front of bridge pillars was sufficiently long according it national regulations (Chapter 3). Since manual VRS classification is only feasible for small road networks, chapter 4 describes in a concise ways how to automatise the classification process. At the end, chapter 5 gives an outlook on different sensing technologies capable of automated roadside environment recognition and safety barrier assessment.

\section{DATA AND METHODS}

At the beginning of a VRS classification process, the systems under surveillance have to be identified by means of visual inspection of the guardrail, the posts and the anchorage. Previous experience with evaluating vehicle restraint systems in Austria revealed that most of the VRS properties necessary to accurately identify a specific system can be obtained from high-resolution images captured from different viewing angles. This information source is used to determine VRS-related attributes including the name of the manufacturer and unique product specifications. Based on the VRS designation, safety-related performance indicators such as containment level, impact severity and working width can be derived from installation manuals and/or product catalogues of the manufacturers.

\subsection{Hardware}

The AIT Austrian Institute of Technology operates a mobile high-performance laboratory called RoadSTAR (Fig. 2), which is used on a regular basis to measure road(side) conditions on primary and secondary road networks. RoadSTAR is equipped with remote sensors (e.g. laser scanners, forward-facing cameras) and a high-precision satellite navigation system to capture a wide range of road surface properties (skid resistance, cracks, surface damages, etc.) and was used in this study to establish VRS pilot inventories on more than $100 \mathrm{~km}$ motorways and expressways to demonstrate the overall feasibility of the developed VRS classification.

To also include VRS in the data collection process, two additional cameras were mounted on a lever arm to record the specific attributes of steel guardrails and concrete barriers during road inspection (Fig. 3). The cameras were mounted in different technical configurations and positions to provide insights on VRS from different angles, i.e. overall view, frontal view of

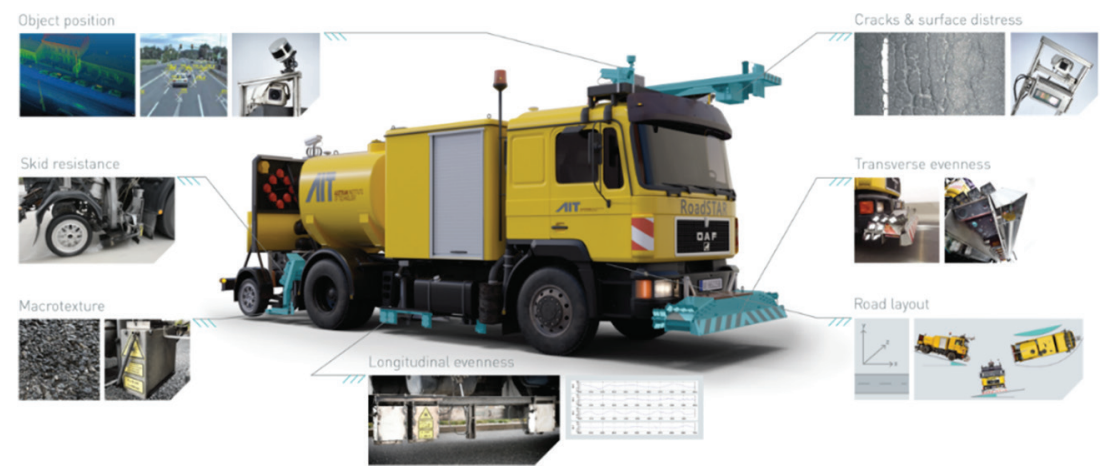

Figure 2: Measurement systems AIT RoadSTAR. 


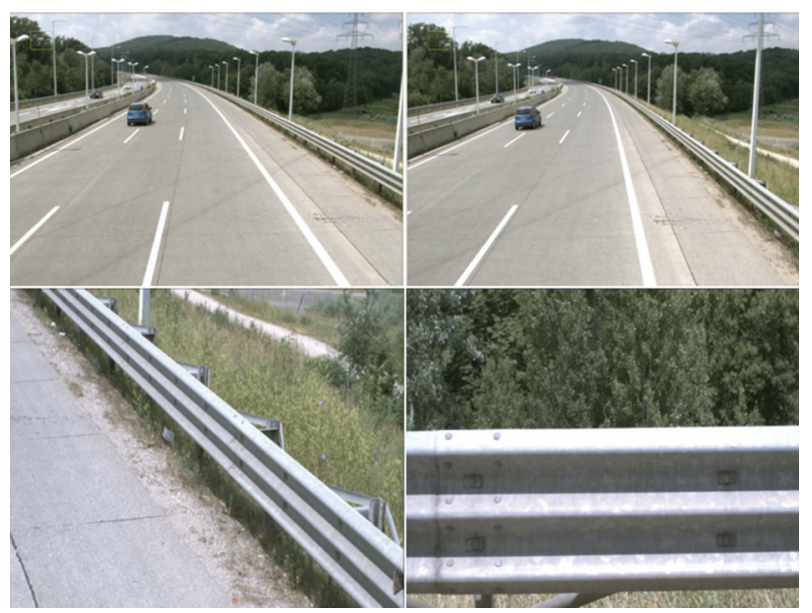

Figure 3: Camera positions: Overall, elevated and frontal view.

the guardrail, view behind the vehicle restraint system in a $45^{\circ}$ angle from an elevated position. In this manner, a close-up view of specific details of the guardrail such as the number of bulges, the profile shape or the bolt pattern could be recorded.

All the cameras met the requirements of industrial standards under outdoor conditions. This includes automatic gain/exposure control, high dynamic range and global shutter. The high precision lenses are able to deliver images with low distortion effects. The two overview cameras facing forward work as a stereoscopic system which also allows 3D measurements. The cameras are distance triggered and coupled with a high precision positioning system.

Post processing with permanent base stations reduces the error of the trajectory to less than $4 \mathrm{~cm}$ under optimal conditions. To obtain high-precision coordinates, the stereoscopic system is linked to an Applanix POSLV 420 inertial navigation system (www.applanix.com), which offers the possibility to digitalize every guard rail or concrete barrier in a GIS application (Fig. 4).

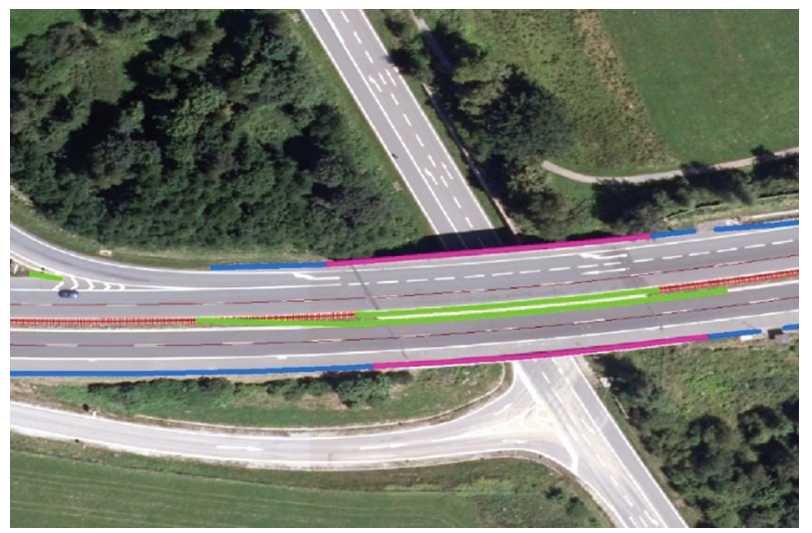

Figure 4: Digitalized and located vehicle restraint systems. 
2.2 Decision tree for guardrail classification

In 2014-2018, scientists from AIT conducted a series of expert interviews and workshops to gain in-depth knowledge on VRS licensed for Austrian roads, leading to the development of an innovative methodology to identify and classify different vehicle restraint systems based on visually detectable attributes. The developed decision tree is only valid for steel barriers, as concrete barriers are characterized in a completely different manner. Hence, a second classification tree for concrete barriers with different decision-making rules needs to be developed, which is not in the focus of this paper. For the purpose of this study, it was sufficient to distinguish between in-situ and ready-mixed concrete barriers.

The VRS classification sequence is based on a step-wise selection procedure (Fig. 5). The decision tree starts with the identification of the cross section based on three attributes: (i) number of bulges, (ii) profile shape and (iii) number of bolts. In total, six decisions sequences are run through before a system identification is possible. Based on the product label, different parameters such as product height, length, impact severity, working width, etc. can be deducted from installation manuals and product sheets. In total, 37 attributes are used for the characterization of different steel barriers.

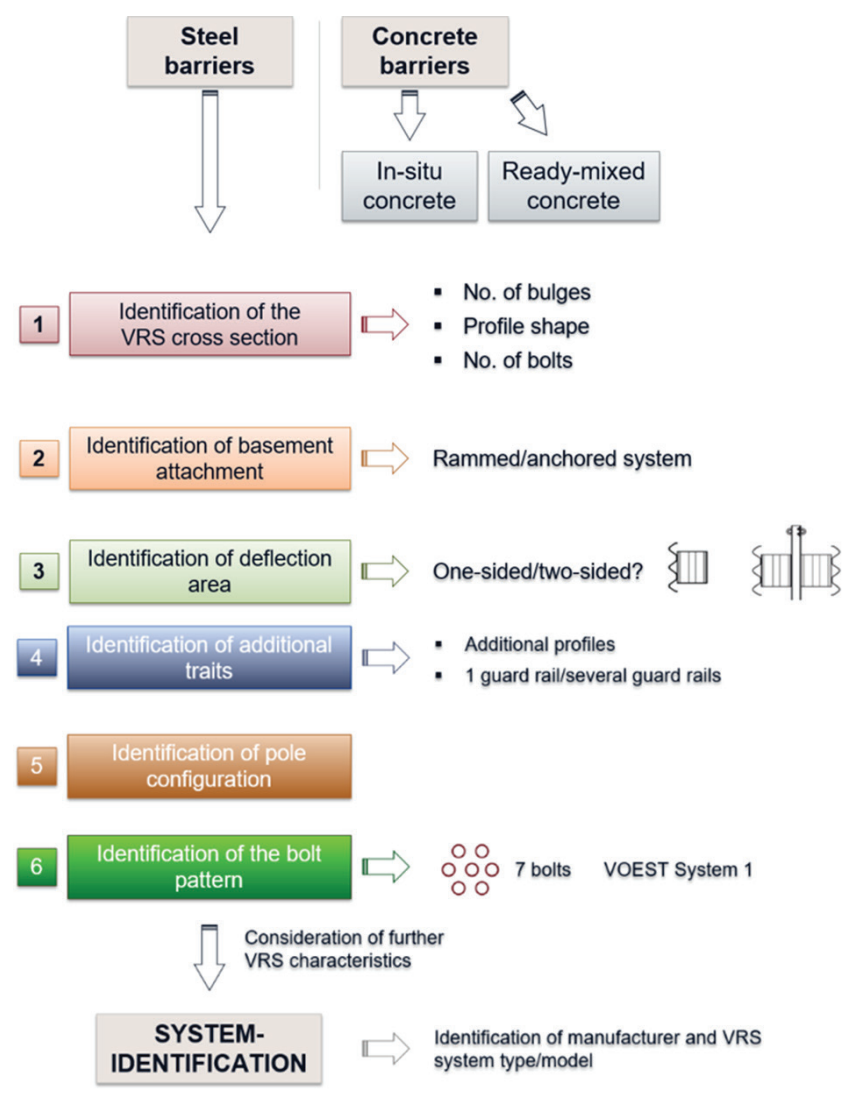

Figure 5: Decision tree for guardrail classification. 


\subsection{Software}

An in-house software solution (RoadVISS - Road Video Information and Survey System) was employed to screen potential VRS candidates based on the (visual) information from the inspection video. The more technical details are entered in the RoadVISS graphical user interface, the better the outcome of the VRS selection process. An optimal solution is reached if only one vehicle restraint system remains in the output window after providing all visually detectable attributes.

In some instances, several plausible vehicle restrain systems remain on the selection screen after entering the input data according to the classification tree. In such a case, additional technical information and construction designs can be requested within the RoadVISS software and summarized in a concise factsheet (Fig. 6).

Experience has shown that comparing the VRS details of the road video with the specifications of the assembly manuals greatly increases the chance of a correct VRS classification; however, that can be cumbersome for the inspection team. In order to reduce the time needed for a correct classification, the information in the VRS factsheets are considered as last resort, since a visual comparison of several pictures is time consuming at the very least.

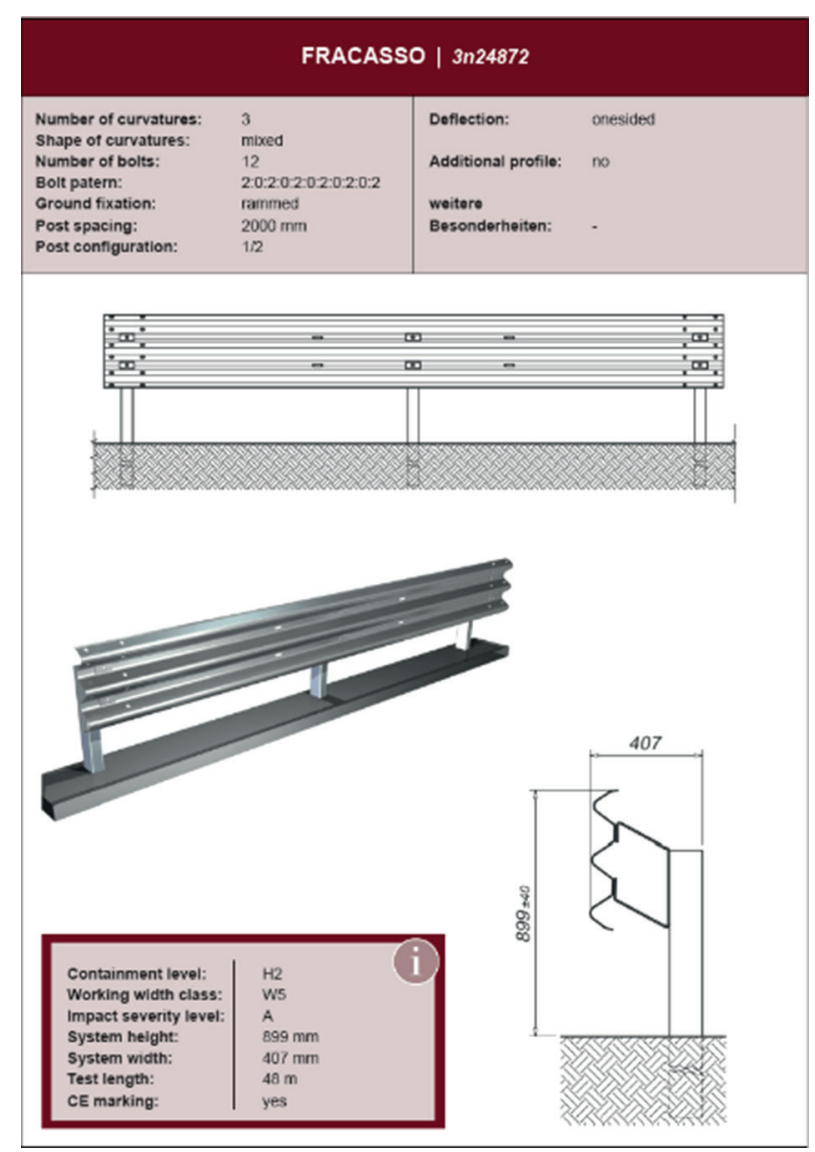

Figure 6: VRS factsheet containing additional information. 


\subsection{GIS-Integration of vehicle restrain systems}

For inventory taking and statistical analyses concerning road safety and asset-related spatial planning activities, the generated VRS information is stored in a Geographical Information System (GIS). Each polyline represents a vehicle restraint system (excluding the beginning and end treatment), whereas the technical details of the system are stored in the attribute list (Table 1).

The 'variable name' denotes the variable tag used in the data set, 'data type' indicates the data type from a data analytical point of view and 'variable description' provides a short explanation of the respective variable. The last column 'unit' contains the physical unit of the variable. Note that the unit [\#] indicates count data (i.e. number of items), while [-] indicates factor variables where no units apply.

Table 1: Attribute list of VRS polylines.

\begin{tabular}{|c|c|c|c|}
\hline $\begin{array}{l}\text { variable } \\
\text { name }\end{array}$ & $\begin{array}{l}\text { data } \\
\text { type }\end{array}$ & variable description & unit \\
\hline vrs_id & string & Master key & {$[-]$} \\
\hline vrs_e & factor & $\begin{array}{l}\text { VRS elements, } 4 \text { levels: Concrete barrier, steel barrier, termi- } \\
\text { nal, unknown. }\end{array}$ & {$[-]$} \\
\hline vrs_d & factor & $\begin{array}{l}\text { Specification of VRS element, } 12 \text { levels (e.g. crash attenua- } \\
\text { tor, terminal) }\end{array}$ & {$[-]$} \\
\hline vrs_ma & string & Manufacturer of the vehicle restraint system & {$[-]$} \\
\hline vrs_pd & string & Product designation of the VRS & {$[-]$} \\
\hline vrs_bn & integer & Number of bulges of the guardrail & {$[\#]$} \\
\hline vrs_ps & factor & Profile shape of the guardrail,3 levels (angular, round, mixed) & {$[-]$} \\
\hline vrs_nb & integer & Number of bolts used for anchorage & {$[\#]$} \\
\hline vrs_hp & string & Hole pattern of the guardrail & {$[-]$} \\
\hline vrs_a & bivariate & $\begin{array}{l}\text { Indicator for type of anchorage, } 2 \text { levels, }(0=\text { rammed, } 1= \\
\text { anchored })\end{array}$ & {$[-]$} \\
\hline vrs_pc & factor & Post configuration of the guardrail, 6 levels (e.g. 1/2, 2/3) & {$[-]$} \\
\hline vrs_dft & bivariate & $\begin{array}{l}\text { Indicator for deflection type, } 2 \text { levels }(0=\text { one-sided, } 1= \\
\text { two-sided })\end{array}$ & {$[-]$} \\
\hline vrs_sp & bivariate & $\begin{array}{l}\text { Indicator for attached sliding profile, } 2 \text { levels }(0=\text { No, } 1= \\
\text { Yes) }\end{array}$ & {$[-]$} \\
\hline vrs_is & factor & Impact severity level of the tested guardrail, 3 levels $(\mathrm{A}, \mathrm{B}, \mathrm{C})$ & {$[-]$} \\
\hline vrs_ww & factor & Working width of the tested guardrail, 8 levels (W1-W8) & {$[-]$} \\
\hline vrs_cl & Factor & $\begin{array}{l}\text { Containment level of the tested guardrail, } 15 \text { levels (e.g. T1, } \\
\mathrm{H} 2, \mathrm{H} 3, \mathrm{H} 4 \mathrm{~b})\end{array}$ & {$[-]$} \\
\hline vrs_dgp & numeric & Distance between guardrail posts & {$[\mathrm{mm}]$} \\
\hline vrs_hg & numeric & Hight of the guardrail & {$[\mathrm{mm}]$} \\
\hline
\end{tabular}




\begin{tabular}{|c|c|c|c|}
\hline vrs_wg & numeric & Width of the guardrail & {$[\mathrm{mm}]$} \\
\hline vrs_tl & numeric & Test length of the guardrail according to EN 1317-2 & {$[\mathrm{m}]$} \\
\hline vrs_ce & bivariate & Indicator for CE marking, 2 levels $(0=$ No, $1=$ Yes $)$ & {$[-]$} \\
\hline vrs_m & factor & $\begin{array}{l}\text { Material of the installed VRS, } 3 \text { levels (concrete, timber, } \\
\text { steel }\end{array}$ & {$[-]$} \\
\hline vrs_bd & bivariate & $\begin{array}{l}\text { Indicator for attached bracket band, } 2 \text { levels, }(0=\text { No, } 1= \\
\text { Yes) }\end{array}$ & {$[-]$} \\
\hline vrs_ag & bivariate & Indicator for additional guardrail, 2 levels, $(0=$ No, $1=$ Yes $)$ & {$[-]$} \\
\hline vrs_st & bivariate & Indicator for attached struts, 2 levels $(0=$ No, $1=$ Yes $)$ & {$[-]$} \\
\hline vrs_h & bivariate & Indicator for attached holm, 2 levels $(0=$ No, $1=$ Yes $)$ & {$[-]$} \\
\hline vrs_tb & bivariate & Indicator for attached tie bars, 2 levels $(0=$ No, $1=$ Yes $)$ & {$[-]$} \\
\hline vrs_df & numeric & Deformation of the VRS during crash testing (EN1317-2) & {$[\mathrm{m}]$} \\
\hline vrs_asi & numeric & Acceleration Severity Index of the tested VRS & - \\
\hline vrs_dd & numeric & Minimum drilling depth of VRS posts & {$[\mathrm{mm}]$} \\
\hline vrs_ce & string & CE-Certificate number & {$[-]$} \\
\hline vrs_rd & date & Date of VRS registration & {$[-]$} \\
\hline vrs_rn & string & Registration number of the tested VRS & {$[-]$} \\
\hline vrs_o & bivariate & $\begin{array}{l}\text { Indicator for anchored terminal within the embankment, }(0= \\
\text { No, } 1=\text { Yes })\end{array}$ & {$[-]$} \\
\hline vrs_sr & bivariate & $\begin{array}{l}\text { Indicator for VRS extended to side roads (bent guardrail } \\
\text { design), }(0 / 1)\end{array}$ & {$[-]$} \\
\hline vrs_p & factor & Post type, 13 levels (e.g. C-Post, H-Post, V-Post, Z-Post) & {$[-]$} \\
\hline vrs_c & string & Comment field for additional information & {$[-]$} \\
\hline
\end{tabular}

\section{RESULTS}

According to the SAVeRS project [12], the length of roadside to be protected by a vehicle restraint system (Length of Need, LON) depends on the size of the hazard and its placement relative to the road edge. The geometrical characteristics of the hazard do not influence the containment class of a VRS, but influence the length of the installation and thereby the installation costs. In most countries, the LON needs to be extended beyond the limits of the hazardous road segment in both directions, i.e. approach and departure, to guarantee a sufficient safety level over the total length of the guardrail.

In Austria, the Research Association for Roads, Railways and Transport (FSV) is responsible for both defining the state of the art in engineering and preparing corresponding guidelines for road and railway transportation systems. The guideline RVS 05.02.31 [11] postulates that VRS must be extended at both ends by $1 / 3$ of the test length (TL) according to EN 1317-2 (Fig. 7).

The developed VRS classification algorithm was tested on $99 \mathrm{~km}$ of Austrian motorways and $11 \mathrm{~km}$ of expressways to evaluate (i) what kind of VRS was installed in front of hazardous road objects and (ii) if the Length of Need of a barrier in front of bridge pillars was sufficiently long according to national regulations. The evaluation yielded that more than $2 / 3$ 


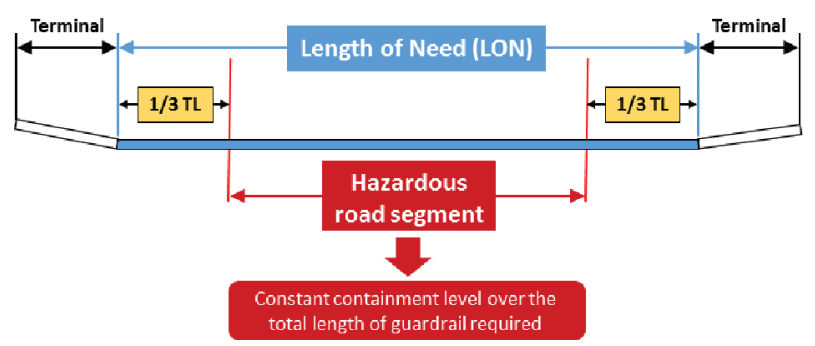

Figure 7: Length of need requirements for vehicle restraint systems.
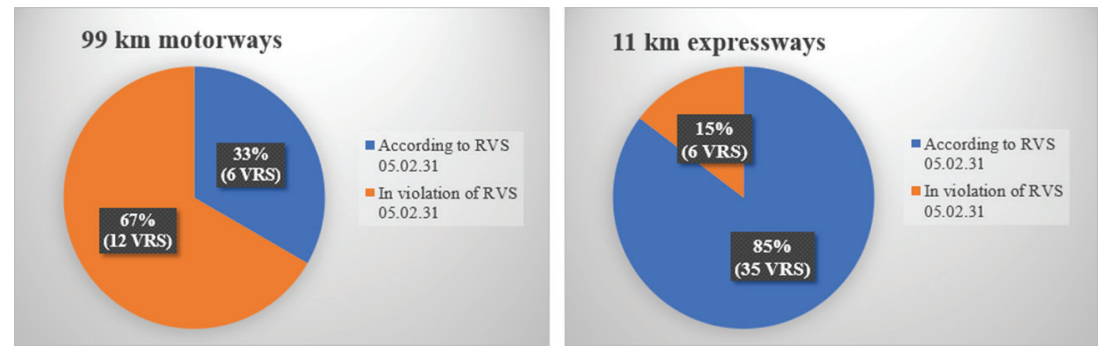

Figure 8: Evaluation of the length of need of guardrails in front of bridge pillars.

(67\%) of the VRS on the investigated motorways and $15 \%$ on expressways did not comply with the RVS 05.02.31 guideline and hence provided an insufficient safety level for vehicles running off the road (Fig. 8).

\section{EMERGING TRENDS FOR AUTOMATED CLASSIFICATION}

Recent developments in machine learning and visual perception open up new ways to characterize roadside infrastructure and their environment in a fully automated manner. In this section we give a concise outlook how this endeavour can be accomplished. From a technology viewpoint, we also characterize which research methodologies are needed to enable a high-speed automated survey under a wide range of illumination conditions.

Vehicle restraint systems are part of the roadside environment. The main goal of a survey is to capture local roadside and VRS characteristics, and to translate these measurements into interpretable measures representing local safety levels for the case of run-off-road accidents. Within the context of a roadside safety management process, such geo-referenced measurements can contribute to monitor and regulate road safety standards at a large geographic scale. Furthermore, given the detailed digital representation of the roadside environment, mathematical modelling and computer simulation can be increasingly used to anticipate potential hazards and to improve on crash-mitigating factors. When complemented with road accident statistical data, such analysis systems can be further used to formulate recommendations for road safety equipment.

The assessment process of VRS is based on multiple key inputs, where state of the art sensory systems and data interpretation can play an important role. Characterizing the geometry and appearance of a safety barrier segment can reveal its type and its condition according to various parameters such as deformations, spatial displacement, parts missing or its reduced integrity. Equally important is the characterization of the roadside environment, in terms of 


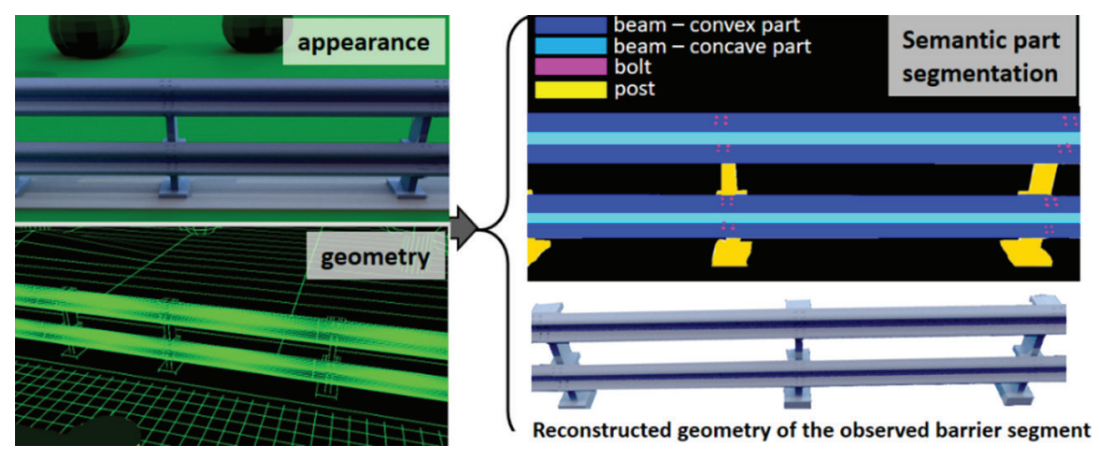

Figure 9: Illustration displaying exemplary appearance and geometry attributes of a VRS.

factors influencing accident severity: road structures (road signs, bridges, abutments, gantries, culverts), obstacles (trees, posts, enclosures) and the characteristics of nearby terrain (slopes, ditches). Recently, novel sensor and analysis technologies are increasingly capable to address the assessment of these observable variables.

An automated survey process is associated with several challenges. The restraint system's large spatial extent and the spatially- and time-varying complexity of observation conditions require fast, robust (in terms of missing or corrupted data) and complementing sensory modalities. Safety barriers of different types often exhibit only small structural differences. To perform an accurate identification of their exact type and to characterize their spatial surrounding in terms of potential collision hazards, geometric and appearance, representations of great discriminative power are needed. Complementary sensory measurements (e.g. depth data and colour images) can well characterize the required geometry and appearance-based attributes. In addition, they introduce a certain redundancy, where information fusion from the individual sensory channels improves the accuracy/robustness of the assessment output.

Geometry and appearance characterization is illustrated in Fig. 9. Geometry data is typically obtained as a depth map or a point cloud, while colour data is provided by digital images. Combining these data channels is often not straightforward, since geometry data 'lives' in a spatial reference frame, whereas images are purely two-dimensional. Geometric sensor calibration provides means to establish a mapping from one reference frame to another; nevertheless, it typically requires a minimally interactive calibration setup prior to the survey process.

\section{OUTLOOK}

In recent years, emerging spatial sensing technologies, especially 3D-laser-scanning [15] and high-resolution stereoscopy [14] [15], provide new ways to capture accurate geometric and appearance information of large-scale environments such as roads and their assets. Both Golovinskiy et al. [16] and Ordóñez et al. [21] propose scientific concepts of mobile laser mapping by Lidar sensors targeting pole-like objects (lamp posts, street trees, traffic lights) in urban environments. While these works do not investigate barrier structures, some of their concepts are applicable to such a scenario. Nevertheless, these systems do not use image information for analysis, as only classification in a voxelized point cloud space is performed.

To our best knowledge, automated image-based digital mapping of roadside barriers is still an unexplored domain. A prototype for automated segmentation and classification of 
roadside infrastructure is proposed by Balali et al. [17]. The work employs a Random Forest classification approach and texton (texture unit) [17] representation, as being the most accurate classification scheme prior to the recently emerged deep distributed representations. Nevertheless, the accomplished low recognition accuracy and segmentation quality makes it impractical for real use. A recent survey on roadside video data analysis via Deep Learning [19] reveals that current learning techniques still do not find a use in safety barrier structures recognition and assessment tasks. The Mapillary Vistas dataset [20] contains annotated image instances for guard-rail structures and for other roadside infrastructure elements, however, no specific VRS assessment solution has been presented based on the dataset yet.

In summary, it can be stated that the field of automated roadside environment recognition and safety barrier assessment offers many exciting future opportunities. The realization of the forgiving roadside concept at a large scale appears to be within reach, pushed by recent technology advances of autonomous driving, depth sensing, machine perception, and by the increasing trend in the large-scale digitization of the world.

\section{REFERENCES}

[1] Thomson, R., Fagerlind, H., Martinez, A.V., Amengual, A., Naing, C.L., Lanner, G., Dupre, G., Bisson, O., Garcia, J., Lopez, F., Hallemann, B., Wink, W., Hoschopf, H., Valtonen, J., Gosse, U., Kelkka, M., Ridder, S.d., Hill, J., \& Van der Horst, R., RISER Roadside Infrastructure for Safer European Roads, Deliverable D06 - European Best Practice for Roadside Design: Guidelines for Roadside Infrastructure on New and Existing Roads, 2006.

[2] Tomasch, E., Sinz, W., Hoschopf, H., Gobald, M., Steffan, H., Nadler, B., Nadler, F., Strnad, B. \& Schneider, F., Required length of guardrails before hazards. In Accident Analysis and Prevention, 43(6), pp. 2112-2120, 2011. https://doi.org/10.1016/j. aap.2011.05.034

[3] Stefan, C., Stütz, R., Tomasch, E., Luttenberger, P. \& Klein, C., A risk-based methodology to assess run-off-road crashes on Austrian motorways - the RISKANT project. In International Journal of Safety and Security Engineering, 6(2), pp. 351-361, 2016. https://doi.org/10.2495/safe-v6-n2-351-361

[4] ASFINAG Service GmbH, Road Safety Programme 2020, Download-Link:https:// www.asfinag.at/media/2970/asfinag_vsb2020_english_einseiter_web_100526.pdf, (accessed 16 May 2019).

[5] OECD/International Transport Forum (ITF), Towards Zero - Ambitious Road Safety Targets and the Safe System Approach, Publisher: Joint Transport Research Centre, Download: https://www.itf-oecd.org/sites/default/files/docs/08towardszeroe.pdf, 2008 (accessed 03 May 2019).

[6] EN 1317-2, Road Restraint Systems - Part 2: Performance Classes, Impact Test Acceptance Criteria and Test Methods for Safety Barriers Including Vehicle Parapets, 2011.

[7] Austrian Standards Institute, Passive Safety of Support Structures for Road EquipmentRequirements and Test Methods, EN 12767, 2011.

[8] American Association of State Highway and Transport Officials (AASHTO), Manual for Assessing Safety Hardware, 2009.

[9] Conference of European Directors of Roads (CEDR), CEDR Transnational road research programme, call 2019 (safety: use of vehicle restraint systems), SAVeRS Defining the Different Parameters Which Can Influence the Need and Selection of VRS, Deliverable D1.1, https://www.cedr.eu/strategic-plan-tasks/research/cedr-call-2012/ call-2012-safety/savers-project-results/, September 2014 (accessed 22 May 2019). 
[10] Conference of European Directors of Roads (CEDR), Forgiving Roadside Design Guide, November 2012. Download-Link:https://www.cedr.eu/download/Publications/2013/T10_Forgiving_roadsides.pdf

[11] Austrian Research Association for Roads, Railways and Transport (FSV), RVS 05.02.31 - Vehicle Restraint Systems, Requirements and Installation, October 2011.

[12] Conference of European Directors of Roads (CEDR), CEDR Transnational Road Research Programme, Call 2019 (Safety: Use of Vehicle Restraint Systems), SAVeRS Guideline for the Selection of the Most Appropriate Roadside Vehicle Restraint System, Deliverable D3. 1 July 2015.

[13] Cho, J. \& Humenberger, M., Fast patchmatch stereo matching using cross-scale cost fusion for automotive applications. IEEE Intelligent Vehicles Symposium, IV 2015, Seoul, South Korea, IEEE, pp. 802-807, 2015.

[14] Zinner, C. \& Humenberger, M., Distributed real-time stereo matching on smart cameras, Fourth ACM/IEEE International Conference on Distributed Smart Cameras, Atlanta, GA, USA - August 31-September 4, 2010, ACM, pp. 182-189, 2010.

[15] Fang, L., Yang, B., Chen, C. \& Fu, H., Extraction 3d road boundaries from mobile laser scanning point clouds, 2nd IEEE International Conference on Spatial Data Mining and Geographical Knowledge Services, ICSDM, pp. 162-165, 2015.

[16] Golovinskiy, A., Kim, V.G. \& Funkhouser, T.A., Shape-Based recognition of 3d point clouds in urban environments, IEEE 12th International Conference on Computer Vision, pp. 2154-2161, 2009.

[17] Golparvar-Fard, M., Balali V. \& de la Garza J.M., Segmentation and recognition of highway assets using image-based $3 \mathrm{~d}$ point clouds and semantic texton forests. Journal of Computing in Civil Engineering, 29(1), p. 04014023, 2012. https://doi.org/10.1061/ (asce)cp.1943-5487.0000283

[18] Shotton, J., Johnson, M. \& Cipolla, R., Semantic texton forests for image categorization and segmentation. IEEE Conference on Computer Vision and Pattern Recognition, pp. 1-8, 2008. https://doi.org/10.1109/cvpr.2008.4587503

[19] Verma, B., Zhang, L. \& Stockwell, D.R.B., Roadside Video Data Analysis: Deep Learning - Deep Learning, Springer, p. 711, 2017.

[20] Neuhold, G., Ollmann, T., Bulò, S.R. \& Kontschieder, P., The mapillary vistas dataset for semantic understanding of street scenes. IEEE International Conference on Computer Vision, ICCV, pp. 5000-5009, 2017.

[21] Ordóñez, C., Cabo, C. \& Sanz-Ablanedo, E., Automatic detection and classification of pole-like objects for urban cartography using mobile laser scanning data. Sensors, 17(7), p. 1465, 2017. https://doi.org/10.3390/s17071465 